\title{
Study on the Collaborative and Interactive Development of the Primary Industry and the Tertiary Industry: Empirical Analysis of the Example of Dujiangyan City
}

\author{
Fuhui Yan (Corresponding author) \\ College of Economic Management, Sichuan Agricultural University \\ No.36, Xing Kang Road, Ya'an 625014, Sichuan, China \\ E-mail: yfh2004127@yahoo.com.cn \\ Qizhi Yang \\ College of Arts and Sciences, Sichuan Agricultural University \\ No.288, Jian She Road, Dujiangyan 611830, Sichuan, China \\ E-mail: yqizhi@scfc.edu
}

The research is supported by the project of "Study on the Collaborative Development of Three Industries in Sichuan Based on the View of the Policy Guidance" of the Sichuan Provincial Philosophical and Social Science Planning Project of 2009 (No. SC08B032).

\begin{abstract}
The industry structure is very important for the economic growth of one country or region, and largely influences the economic boom and future development degree of this country or region. In some regions, because of the resource gift, only two industries are mainly developed in the industry structure, and there are few researches about the interactive development of these two industries. Taking the city of Dujiangyan as the example, the quantity economic model is established in this article, and the regional economic development mode giving priority to the primary industry and the tertiary industry is discussed, which could be used as references for the economic development of same regions.
\end{abstract}

Keywords: Primary industry and tertiary industry, Collaborative interaction, Grey correlation analysis

\section{Introduction}

The industry structure is very important for the economic growth of one country or region, and largely influences the economic boom and future development degree of this country or region. In China, the industry structure is mainly composed by the primary industry, the secondary industry, and the tertiary industry, so most scholars studied the interactive or collaborative development of these three industries (Zhao, 2009 \& Dong, 2008 \& Ren, 2005 \& Jia, 2001\& Qiao, 2004 \& Liu, 2002 \& Ouyang, 2006 \& Yang, 2003), and some scholars studied the trans-regional industry collaborative development for the harmonious development of regional economies (Yang, 2009). But in some regions, because of the influences from historical factor, natural resources, political and economic factors, only two industries have been mainly developed, and the researches about how to realize the interaction of these two industries are vary rare in the academe. For example, the city of Dujiangyan is the main water supply position of Chengdu, so this place is not suitable to develop the scale industry enterprises, and Dujiangyan has two world cultural heritages, i.e. the Dujiangyan and the Qingcheng Mountain, with comfortable climate, and it is optimal place to develop the tourism industry. At the same time, Dujiangyan is the granary of Chengdu, and the agriculture is the important industry of Dujiangyan. How to realize the interactive development of the primary industry and the tertiary industry, i.e. the agriculture and the tourism, promote the local economy, and establish the durative income increasing mechanism for farmers is very important for Dujiangyan. The quantitative economic model is established in this article to discuss the economic development mode of Dujiangyan, which could be used as references for the economic development of same regions. 


\section{Interactive development model of the primary industry and the tertiary industry in the Dujiangyan City}

\subsection{Variable selection explanation and data source}

\subsubsection{Explanation of variable selection}

Not only the proportion relationship between the primary industry and the tertiary industry is the factor to influence the optimization degree of the industrial structure, but also the proportion of various factors in the industry will largely influence the industrial structure. By analyzing the association strengths among various factors, the interactive and collaborative relationship between the primary industry and the tertiary industry will be analyzed in this article. So the production value structure, the employment structure, and the fixed asset structure with stronger operation of the primary industry and the tertiary industry will be selected as the variables. The production value structure of the industry is the proportion relationship between the primary industry and the tertiary industry, and the production value proportion of the primary industry $=$ the added value of the primary industry/ GDP, and the production value proportion of the tertiary industry = the added value of the tertiary industry/ GDP. The employment structure of the industry means the distribution, the composing, and the association of the labor force in various departments in the primary industry and the tertiary industry, and the employment proportion of the primary industry $=$ the employees of the primary industry/ the total employees of the whole city, and the employment proportion of the tertiary industry = the employees of the tertiary industry/ the total employees of the whole city. The fixed asset investment proportion of the primary industry $=$ (the fixed asset investment of the whole city/ GDP)* (the production value of the primary industry/ GDP), and the fixed asset investment proportion of the tertiary industry $=$ (the fixed asset investment of the whole city/ GDP)* (the production value of the tertiary industry/GDP).

\subsubsection{Data source}

According to the requirements of the model, the data in this article mainly comes from the Sichuan Statistic Yearbook and the Dujiangyan City Statistic Yearbook from 1999 to 2007.

\subsection{Model}

The model in this article is Professor Deng Julong's grey system model which was first put forward in his paper of "The Control of the Grey System" issued in 1982. He divided the information into the white, the black, and the grey, and the white represents the information is completely definite, and the black represents the information is completely indefinite, and the grey represents that the information is in these two states. In this theory, the indefinite system with "small sample" and "poor information" in which part information is known and part information is unknown is the research object, and the known information is utilized to seek the valuable influencing factors to effectively control the similar behaviors. The grey association analysis is a multiple-factor statistic analysis method, and it compares the geometric relationship of relative statistic data in various periods of the development and change system, and confirms the advantages and disadvantages influencing the development of the system by the quantitative description and the comparison analysis. The basic idea of the grey association analysis is to analyze and compare the geometric forms of several curves, i.e. it thinks that the geometric forms are more similar, the tendencies of the development and the change are closer, and the association degree is stronger.

The establishment of the model includes following steps.

Step 1. Confirm the line sequence of the analysis data. Establish the reference sequence (mother sequence) of the original data and the compared sequence (son sequence).

Reference sequence: $\mathrm{X}_{0}=\left\{x_{0}(1), x_{0}(2), x_{0}(3), \cdots x_{0}(n)\right\}$

Compared sequence: $\mathrm{X}_{1}=\left\{x_{1}(1), x_{1}(2), x_{1}(3), \cdots x_{1}(n)\right\}$

$\mathrm{X}_{2}=\left\{x_{2}(1), x_{2}(2), x_{2}(3), \cdots x_{2}(n)\right\}$

......

$\mathrm{X}_{m}=\left\{x_{m}(1), x_{m}(2), x_{m}(3), \cdots x_{m}(n)\right\}$

Step 2. Normalize and compute the original data by the average value method or the original value method.

Step 3. Compute the difference sequence $\Delta_{i}$ between the reference sequence $\mathrm{X}_{0}(k)$ and the compared sequence $\mathrm{X}_{m}(k)$. 


$$
\begin{aligned}
& \Delta_{i}=\left|\mathrm{X}_{0}(k)-\mathrm{X}_{m}(k)\right|=\left(\Delta_{1}(1), \Delta_{1}(2), \cdots, \Delta(n)\right) \\
& \Delta_{i}=\left|\mathrm{X}_{0}(k)-\mathrm{X}_{m}(k)\right|=\left(\Delta_{2}(1), \Delta_{2}(2), \cdots, \Delta_{2}(n)\right) \\
& \cdots \cdots \\
& \Delta_{i}=\left|\mathrm{X}_{0}(k)-\mathrm{X}_{m}(k)\right|=\left(\Delta_{n}(1), \Delta_{n}(2), \cdots, \Delta_{n}(n)\right)
\end{aligned}
$$

Step 4. Compute the minimum value and the maximum value.

Step 5. Compute the association coefficient. The computation formula is

$$
r(x(k), x(k))=\frac{\min _{i} \min _{k}\left|x_{0}(k)-x_{i}(k)\right|+\xi \max _{i} \max _{k}|x(k)-x(k)|}{\left|x(k)-x_{i}(k)\right|+\xi \max _{i} \max _{k}\left|x_{0}(k)-x_{i}(k)\right|}
$$

Where, $\xi$ is the resolution ratio in $0 \sim 1.0 .5$ is selected in this article.

Step 6. Compute the association degree. The computation formula is

$$
r\left(\mathrm{X}_{0}, \mathrm{X}_{i}\right)=\frac{1}{N} \sum_{i=1}^{N} r\left(x_{0}(k), x_{i}(k)\right)
$$

2.3 Grey correlation analysis of the collaborative degree of the primary industry and the tertiary industry in Dujiangyan City

The industrial structure of one region is denoted by the proportion of the industrial added value in GDP, or the proportion of the employment amount of the industry in the total employment amount, or the fixed asset investment proportion, which indicates that the reasonable degree of the production value structure or the employment structure or the fixed asset structure will influence the industrial structure optimization degree of this region. By computing the correlation degree between the production value structure and the employment structure and the fixed asset structure in the primary industry and the tertiary industry of Dujiangyan, the production value structure is the reference sequence, and the employment structure and the fixed asset investment structure are the compared sequence. By judging the collaborative degree of the industrial development of Dujiangyan, the actual situation of Dujiangyan could be adjusted correspondingly, and the collaborative development of the primary industry and the tertiary industry could be promoted, and the industrial structure could be further optimized, and the economy could be further developed.

2.3.1 Grey correlation analysis of the collaborative degree of the primary industry

The production value proportion, the employment proportion, and the fixed asset investment proportion of the primary industry of Dujiangyan are seen in Table 1.

According to the solution approaches of the grey association degree, the result could be computed as follows.

$$
\begin{gathered}
r_{01}=\frac{1}{N} \sum_{i=1}^{N} r\left(x_{0}(k), x_{i}(k)\right)=0.858352 \\
r_{02}=\frac{1}{N} \sum_{i=1}^{N} r\left(x_{0}(k), x_{i}(k)\right)=0.646239
\end{gathered}
$$

Above result shows that the association degree between the production value structure and the employment structure of the primary industry is 0.858352 and the association degree between the production value structure and the fixed asset investment structure of the primary industry is 0.646239 . The association degree of the former is bigger than the association degree of the latter, which indicates that the labor force is relatively important in the development of the primary industry.

2.3.2 Grey correlation analysis of the collaborative degree of the tertiary industry

The production value proportion, the employment proportion, and the fixed asset investment proportion of the tertiary industry of Dujiangyan are seen in Table 2.

According to the solution approaches of the grey association degree, the result could be computed as follows.

$$
r_{01}=\frac{1}{N} \sum_{i=1}^{N} r\left(x_{0}(k), x_{i}(k)\right)=0.956594
$$




$$
r_{02}=\frac{1}{N} \sum_{i=1}^{N} r\left(x_{0}(k), x_{i}(k)\right)=0.654945
$$

Above result shows that the association degree between the production value structure and the employment structure of the primary industry is 0.956594 and the association degree between the production value structure and the fixed asset investment structure of the primary industry is 0.654945 . The association degree of the former is bigger than the association degree of the latter, which indicates that the labor force is relatively important in the development of the tertiary industry.

\section{Conclusions}

By the grey association analysis, the association degree of the production value structure and the employment structure is higher than the association degree of the production value structure and the fixed asset investment. From Figure 1 and Figure 2, the proportions of the fixed asset investment of the primary industry and the tertiary industry is lower than the production value proportion of the primary industry and the tertiary industry, and much lower than the employment proportion of the labor force in the primary industry. That indicates that the collaborative development of Dujiangyan mainly depends on the reasonable change of the employment structure, i.e. the rationalization of the employment structure is the effective approach of the collaborative interaction of the primary industry and the tertiary industry, or else, the optimization of the industrial structure will be largely blocked.

Second, the fixed asset investment will influence the collaboration of the primary industry and the tertiary industry to some extent, and the investment direction should be carefully selected. Relative departments should invest to the collaborative development of the primary industry and the tertiary industry, and incline the investment to the infrastructure.

The collaboration of the primary industry and the tertiary industry of Dujiangyan is related not only with the employment proportion and the fixed asset investment, but also with other factors which are not been listed in this article. And which industry in the primary industry and the tertiary industry will influence the industrial collaboration most has not been explained in detail, so the research about the industrial collaboration of Dujiangyan should continue to collect data and select more industries for the association degree analysis of the primary industry and the tertiary industry, in order to find the dominant industries and sunrise industries and realize the dynamic collaboration.

\section{Policy advices}

From above conclusions and problem analysis, the existing industrial structure of the primary industry and the tertiary industry of Dujiangyan has not achieved Chenery's standard industrial structure, and the industrial collaboration degree is still bad, and to better realize the collaborative development of the primary industry and the tertiary industry, following advices should be noticed.

First, to better realize the collaboration of the industrial structure of the primary industry and the tertiary industry of Dujiangyan, the employment problem should be first noticed. The government should enhance farmers' quality by various ways, increase the training times, develop the labor-intense industries, and solve farmers' employment problem. After the Earthquake, the primary industry of Dujiangyan still has large numerous surplus labor forces, and when the government transfers the surplus labor force from the village to the city, it should strengthen the infrastructure construction of the agriculture, implement the industrialization of the agriculture, and develop the agriculture with special characteristics, for example, developing five specially advantageous agricultures including Chinese gooseberry, tea-leaf, cold-water fish, pig, vegetable and domestic fungus to further enhance the production of the primary industry. For the tertiary industry, according to the actual situation of Dujiangyan, the government should largely develop the modern service industry such as the tourism, especially the tourism industry with special characteristics such as the rural tourism, driving the development of relative industries and extending the industrial chain, and providing more employment posts for the employees in the primary industry and the tertiary industry. At the same time, the ecological agriculture should be recovered and developed, and depending on the production base of five large advantageous farm products, the government should actively develop the agricultural leisure and tourism and promote the collaborative and interactive development of the primary industry and the tertiary industry. The government should also perfect and issue relative policies about the finance and the taxation, strengthen the support degree to the development of the primary industry and the tertiary industry, encourage returned farm workers to create their own businesses, create conditions and provide attractive conditions to attract foreign investors to invest in Dujiangyan, and drive the quick development of the primary industry and the tertiary industry of Dujiangyan. 
Second, the government should make the scientific planning of the collaborative development of the primary industry and the tertiary industry of Dujiangyan. The planning could provide convenient conditions for the primary industry and the tertiary industry of Dujiangyan, confirm the development idea, and ensure the collaboration of the primary industry and the tertiary industry. At the same time, the natural resource, the environment resource, and the human resource of Dujiangyan should be utilized to develop the special and advantageous industries, and drive the harmonious and healthy development of relative industries by the tourism industry.

Third, for the collaborative development of the primary industry and the tertiary industry of Dujiangyan, the government should strengthen the cooperation with relative cities and counties, and fully utilize the present software and hardware establishments by the industrial alliance, and create the new situation of the industrial collaboration and development of Dujiangyan together.

\section{References}

Dong, Chun \& Da, Jie. (2008). Study on the Collaborative Development of Three Industries in Sichuan. Reform of Economic System, No.8.

Jia, Xiaofeng. (2001). Historical Evolvement and Trend of Three Industrial Structure in Our Country. China Statistics, No.9.

Liu, Wei \& Li, Shaorong. (2002). Industrial Structure and Economic Growth. Industrial Economy of China, No.5.

Ouyang, Jianguo \& Yu, Fugong. (2006). Dynamic Relationship among Three Industries' Mutual Influence. Shanghai Economic Review, No.10.

Qiao, Weiguo \& Zhou, Weifeng. (2004). A Explanation of Industrial Structure Characteristics in China. Quantitative \& Technical Economics, No.11.

Ren, Gangju \& Xue, Xiangji. (2005). Study on the Interactive Relation of the Development Speeds of Three Industries. Statistics and Decision, No.19.

Yang, Jie, Wang, Yan \& Liu, Xiao. (2009). To Explore on the Way of Synergic Developing of the Regional Industries in Beijing-Tianjin-Hebei. Value Engineering, No.4.

Yang, Jirui. (2003). Consideration about Implementing the Strategy of "Interacting Three Industries and Integrating Urban and Rural Economies". Rural Economy, No.2.

Zhao, Kai. (2009). Research on the Interactions between the Tertiary Industry and the Primary/Secondary Industries in Henan Province. Economic Survey, No.2.

Table 1. The production value proportion, the employment proportion, and the fixed asset investment proportion in the primary industry

\begin{tabular}{|c|c|c|c|}
\hline \multirow{2}{*}{ Year } & Primary Industry & Primary Industry & Primary Industry \\
\cline { 2 - 4 } & Production Value Proportion & Employment Proportion & Fixed Asset Investment Proportion \\
\hline 1999 & 0.217404253 & 0.457332948 & 0.046709317 \\
\hline 2000 & 0.172842852 & 0.455692488 & 0.040330106 \\
\hline 2001 & 0.158188059 & 0.452148148 & 0.046682048 \\
\hline 2002 & 0.146025986 & 0.395899509 & 0.049909165 \\
\hline 2003 & 0.124917148 & 0.363078639 & 0.046154095 \\
\hline 2004 & 0.117901751 & 0.350762527 & 0.045779274 \\
\hline 2005 & 0.139195773 & 0.324661534 & 0.072598553 \\
\hline 2006 & 0.129987838 & 0.310750719 & 0.090749824 \\
\hline 2007 & 0.132537284 & 0.288793103 & 0.103287541 \\
\hline
\end{tabular}

Data source from: Sichuan Statistical Yearbook 
Table 2. The production value proportion, the employment proportion, and the fixed asset investment proportion in the tertiary industry

\begin{tabular}{|c|c|c|c|}
\hline \multirow{2}{*}{ Year } & Tertiary Industry & Tertiary Industry & Tertiary Industry \\
\cline { 2 - 4 } & Production Value Proportion & Employment Proportion & Fixed Asset Investment Proportion \\
\hline 1999 & 0.437898795 & 0.330633497 & 0.094082582 \\
\hline 2000 & 0.449707678 & 0.331866197 & 0.10493207 \\
\hline 2001 & 0.455673199 & 0.32 & 0.134471328 \\
\hline 2002 & 0.439279843 & 0.35114063 & 0.150138279 \\
\hline 2003 & 0.43400892 & 0.361405466 & 0.160356596 \\
\hline 2004 & 0.566234943 & 0.358115468 & 0.219859538 \\
\hline 2005 & 0.504390866 & 0.384125299 & 0.263068672 \\
\hline 2006 & 0.506301681 & 0.400732409 & 0.353469904 \\
\hline 2007 & 0.498412433 & 0.40872211 & 0.388417454 \\
\hline
\end{tabular}

Data source from: Sichuan Statistical Yearbook

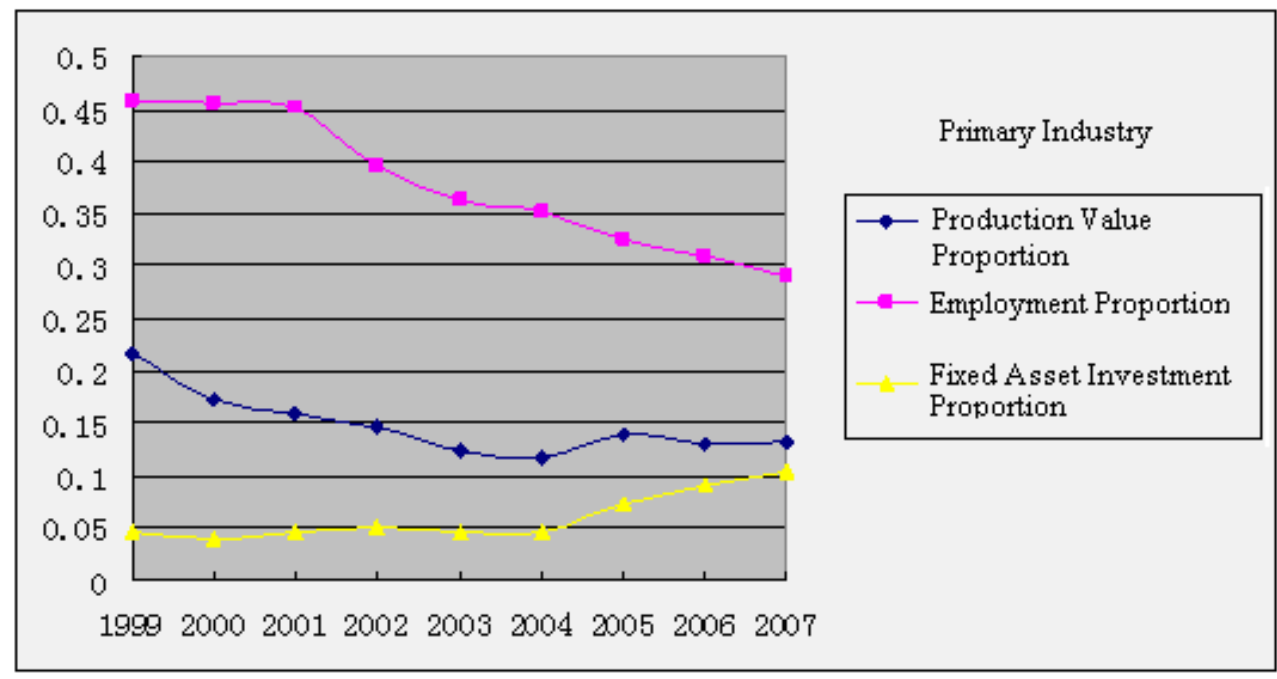

Figure 1. Figure of the Production Value Proportion, the Employment Proportion, and the Fixed Asset Investment Proportion in the Primary Industry

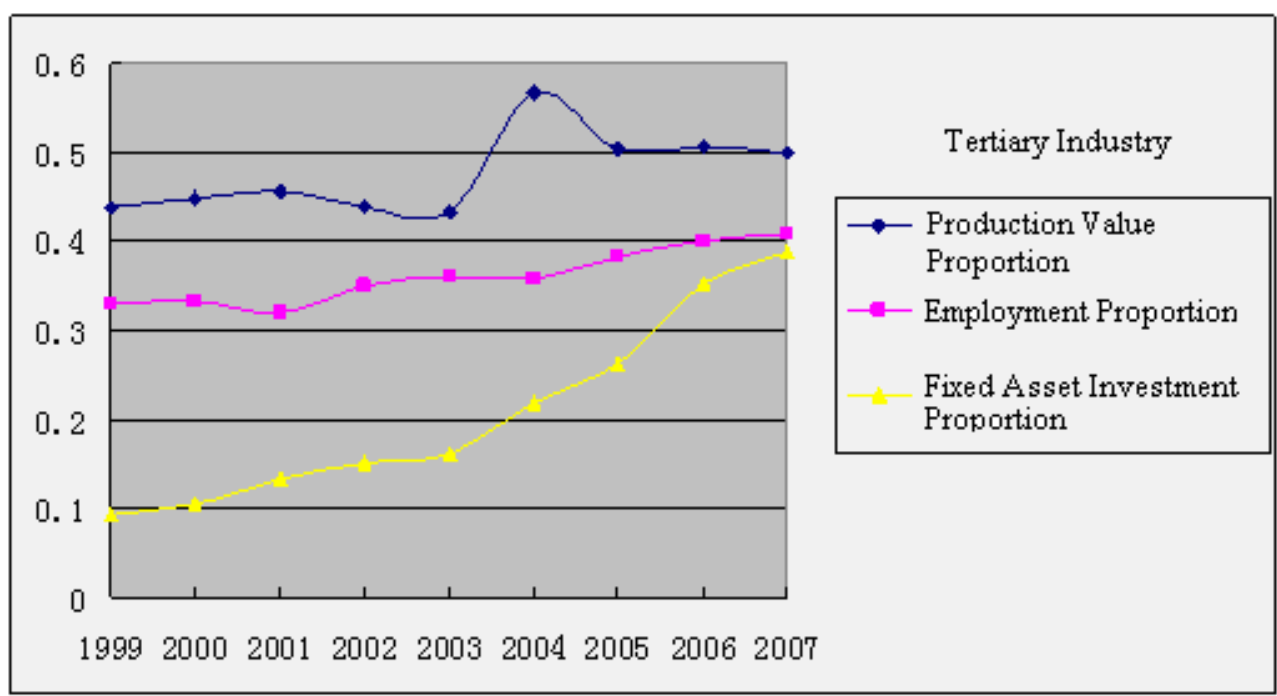

Figure 2. Figure of the Production Value Proportion, the Employment Proportion, and the Fixed Asset Investment Proportion in the Tertiary Industry 